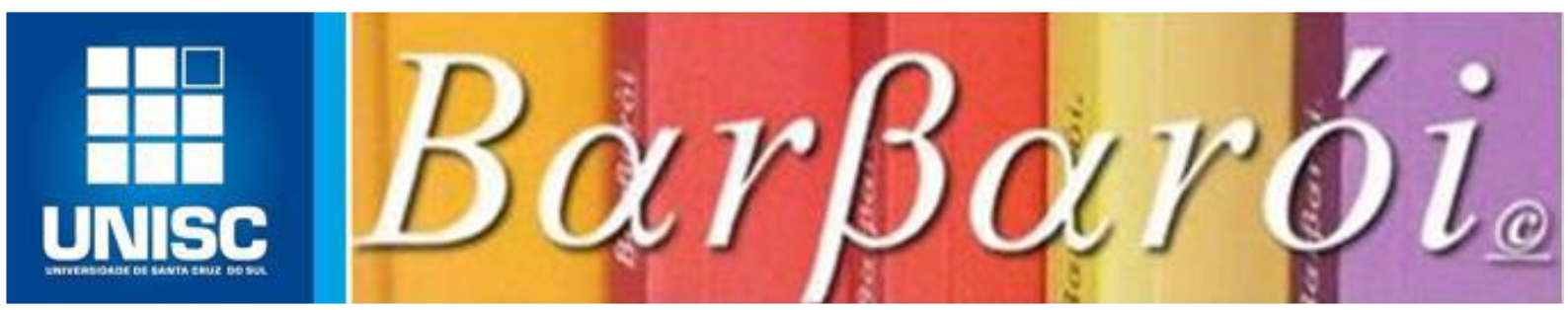

\title{
ESTRATÉGIA DA SAÚDE DA FAMÍLIA: A DIMENSÃO ARTICULADORA DO TERRITÓRIO
}

\author{
DOI: http://dx.doi.org/10.17058/barbaroi.v0i0.14643

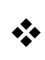

Gláucio Jorge Souza

Universidade do Vale do Paraiba - UNIVAP - Brasil

Cilene Gomes

Universidade do Vale do Paraíba - UNIVAP - Brasil

Valéria Regina Zanetti

Universidade do Vale do Paraiba - UNIVAP - Brasil

$*$

\section{Resumo}

O estudo, de caráter descritivo e analítico, visa examinar o processo de implantação da Estratégia de Saúde da Família (ESF) no Brasil efetivando serviços de saúde pública no contexto do Sistema Único de Saúde (SUS). Para tal, utilizou-se a revisão de literatura e dados do Ministério da Saúde. Nesse contexto, destaca-se o valor da prática assistencial multi e interdisciplinar e da territorialização e do mapeamento como instrumentos importantes para a constante avaliação do programa, o planejamento das ações intersetoriais e a gestão das unidades e equipes da ESF, pois possibilitam o conhecimento das populações a serem atendidas e das condições sócio territoriais e de saúde das mesmas. Observa-se em 20 anos um aumento de Agentes Comunitários de Saúde (ACS) e das equipes da ESF em todas as regiões do país, mas a reorganização da Atenção Básica $(\mathrm{AB})$ em saúde continua sendo um dos maiores desafios para o aperfeiçoamento da gestão e provisão dos serviços, e por isso, para as equipes de profissionais envolvidas.

Palavras-chave: Agente Comunitário de Saúde; Territorialização; Saúde Coletiva.

\section{Introdução}

A consolidação da Estratégia da Saúde da Família (ESF) é um processo complexo que envolve a interdisciplinaridade e a constante avaliação das práticas profissionais. Observa-se, hoje, um aumento de publicações sobre políticas públicas, embora ainda sejam poucos os estudos sobre a heterogeneidade da implantação da ESF no cenário nacional (FARIA, 2003). 
A Constituição Federal (CF) brasileira de 1988 é um marco político-institucional do estado democrático de direito na busca de justiça social. Transformações no Estado e na sociedade eram esperadas, almejando-se o fortalecimento de valores democráticos em todos os âmbitos da vida social (SILVA, 2012). Na década de 1980, a Reforma Sanitária se inseriu no contexto de luta social pela redução das desigualdades, que resultou na CF/1988. A partir dela, surgem novas leis que darão origem e amparo a uma nova geração de políticas públicas (ROSA, SAES, ABULEAC, 2012).

Pensou-se a política pública com a perspectiva de atender às necessidades sociais e transformar a vida dos cidadãos. A implementação e a manutenção da política pública seriam de responsabilidade do Estado e sua efetivação se daria por meio de processos e decisões envolvendo a participação de diferentes setores, órgãos públicos, organizações e agentes da sociedade diretamente ligados às ações e peculiaridades de cada política pública (SOUZA, 2006).

Com a CF e a Lei Federal 8080, determinaram-se os princípios norteadores da política e organização dos serviços de saúde em distintas esferas governamentais, por meio de um sistema de atenção integrada, visando oferecer saúde à sociedade em geral, o que resulta na criação do Sistema Único de Saúde (SUS) (BRASIL, 1990).

Embora se contemplasse, na época, os efeitos positivos do novo modelo de atenção, o SUS ainda hoje não conseguiu suprir as múltiplas e diferenciadas demandas da saúde pública e de superar completamente o sistema pré-existente, considerado caótico e deficitário, com a assistência e atenção à saúde voltada só para a doença e os aspectos biológicos do indivíduo, centrada na figura de um único profissional, o médico.

Ainda assim, o Conselho Nacional de Secretários de Saúde (CONASS) aponta que, com o SUS, esse cenário começa a mudar. O foco na atenção à saúde está agora na prevenção, promoção, reabilitação e educação dos cidadãos. As intervenções na saúde se dão em menor tempo, com o início da prática profissional antes mesmo do adoecimento do indivíduo, e este passou a ser contemplado como um ser biopsicossocial (CONASS, 2011).

A sociedade brasileira passa a contar com uma rede de serviços mais organizados e distribuídos por todo o território. A nova política pública de saúde orienta-se por novos princípios até então não praticados. Destacam-se: a universalidade do acesso a todos os cidadãos; a equidade do atendimento conforme as necessidades de cada comunidade ou indivíduo; e a integralidade, com o atendimento do indivíduo em todas as suas necessidades. Com isso, o conceito de saúde se amplia.

Em 1991, o Ministério da Saúde cria o Programa de Agentes Comunitários de Saúde Barbarói, Santa Cruz do Sul, n. 56, p.<141-163>, jan./jun. 2020 
(PACS), tendo o protagonismo do Agente Comunitário de Saúde (ACS), com a função principal de intermediação entre a provisão pública dos serviços de atenção básica e a comunidade adstrita em seu território de atuação. A dimensão sócio territorial de vida das populações beneficiárias torna-se um dado central da implementação da política. Objetiva-se uma assistência mais próxima e fundada no conhecimento dos beneficiários e de sua condição de vida, com o fim de facilitar a tomada de decisão antes do adoecimento (FARIA et al., 2010).

No geral, apesar de suas deficiências, constata-se que o programa tem trazido efeitos positivos na saúde das populações, sobretudo as mais carentes, com melhoria de indicadores. Dentre outros, notou-se aumento da cobertura dos pré-natais e melhoria no aporte nutricional. O programa contribuiu significativamente para a elaboração de novas estratégias originando, então, o Programa da Saúde da Família (PSF) (SOUSA, 2009).

O PSF foi instituído pelo Governo Federal em 1994, para substituir o modelo tradicional de atenção à saúde. Nessa nova modalidade, além dos ACS, a equipe compõe-se por diferentes profissionais, cada um com atribuições e atividades inerentes à sua categoria: médicos, enfermeiros, técnicos e auxiliares de enfermagem, dentistas, agentes de consultório dentário (ACD), e inclusive psicólogos e assistentes sociais. Todos respondem pelo território definido para atuação e pela população que nele habita (SOUSA, 2009).

O objetivo do programa foi orientar a prática assistencial centrada na atenção ao indivíduo como parte da família que, por sua vez, faz parte de uma comunidade. A assistência à saúde torna-se contextual e a ação continuada, quando então o PSF tornou-se a Estratégia de Saúde da Família (ESF), com a perspectiva de garantir permanência ao processo de reorganização da atenção básica.

Nesse artigo, objetivou-se a compreensão da Estratégia da Saúde da Família (ESF) - na sua condição como programa para a reorganização e readequação do serviço de saúde no nível da atenção básica. Buscou-se também refletir sobre a sua implementação e contribuição para a saúde da população e efetivação do SUS. Além disso, buscou-se elaborar um perfil da experiência da ESF em território brasileiro.

\section{Material e método}

Para realização desse estudo, procedeu-se a uma revisão da literatura para compreensão conceitual do objeto em estudo. As referências selecionadas foram as da produção científica sobre a implementação da ESF, a partir dos descritores de assuntos em Ciências da Saúde (DECs) e das seguintes palavras-chave: Agente Comunitário de Saúde; Territorialização; Saúde 
Coletiva. Foram consultados artigos científicos publicados em periódicos indexados nas bases de dados Lilacs, Scielo, Documentos Oficiais (Leis, Decretos, Portarias), além de outros trabalhos científicos, indexados no período de 1988 a 2018, no idioma português do Brasil. Foram excluídos os artigos que não tinham relação com o processo de implantação da ESF e os publicados fora do período em foco.

O estudo, de caráter descritivo, explora, nas considerações finais, os potenciais de análise futura da política pública de saúde e, particularmente da ESF, em sua condição de política territorial preocupada com a universalização, equidade e regionalização dos serviços, e com a descentralização da gestão. Com isso, pretende-se reafirmar a hipótese de que, a importância da dimensão territorial da política articula a possibilidade real de efetivação do direito à saúde, a intersetorialidade da ação pública e a interdisciplinaridade do conhecimento.

\section{A estratégia da saúde da família: contexto e implantação}

A $8^{a}$ Conferência Nacional de Saúde (CNS), realizada em 1986, e a CF/1988 são considerados marcos de conquista de diversos direitos à população e do retorno do país ao regime democrático. A CNS contou com a participação ativa da população. Mais de cinco mil representantes de todos os segmentos da sociedade civil saíram às ruas para discutir um novo modelo de saúde para o país. A CF/1988 transformava a saúde em direito de cidadania e dever do Estado, dando origem ao processo de criação de um sistema público, universal e descentralizado de saúde, o SUS. A partir de então, iniciaram-se debates sobre a saúde coletiva e a atenção básica, em vista de melhoria das condições de vida dos cidadãos; o avanço da democratização e dos direitos universais.

Os movimentos sociais e as experiências de alguns deles junto às comunidades foram propulsores de discussões que desenharam, em escala nacional, o Programa de Agentes Comunitários de Saúde (PACS) e o Programa de Saúde da Família (PSF), que têm sido implantados em território brasileiro como modelo de reorganização das políticas de saúde desde, aproximadamente, a década de 1990 (PESSOA, 2005).

O Programa de Agentes Comunitários de Saúde (PACS) foi um dos primeiros programas instituídos após a criação do SUS, considerado um programa de transição para o PSF, criado no Brasil em 1994, com os mesmos princípios de universalização dos serviços e integralidade da assistência para reorientar, reorganizar e aperfeiçoar o modelo assistencial em saúde então vigente. Visava-se mudar a concepção do processo de cuidar, atribuindo importância ao indivíduo como sujeito com necessidades e direitos e à multi-profissionalização das equipes. Dalpiaz e Stedile (2011, p. 3) ressaltam que "estender a cobertura assistencial às Barbarói, Santa Cruz do Sul, n. 56, p.<141-163>,jan./jun. 2020 
áreas mais pobres e de risco visam à equidade”, o terceiro princípio do SUS, imprescindível para a "garantia de direitos".

Em 2006, o PSF deixou de ser um programa, passando a ser denominado ESF, com ação constante e permanente. No mesmo ano, como forma de consolidação do SUS, foi implantado o Pacto pela Saúde, priorizando o fortalecimento da Atenção Básica (AB) e, por conseguinte, a ESF seguiu ações na mesma lógica da atenção básica ou primária, com foco na prevenção e promoção da saúde.

Há na literatura evidências de que a ESF, além de seguir estratégias próprias, apoia-se nos mesmos pilares que norteiam o SUS e a AB. Todos se constituem em ações que garantem o que a Constituição preconiza, acessibilidade e qualidade dos serviços. Sendo uma estratégia que compõe e legitima o SUS, a ESF se define por meio de práticas em saúde desenvolvidas em contextos territoriais e populacionais específicos e, ainda, pela participação ativa no que tange à gestão deste programa (CONASS, 2011).

Implantar e manter a qualidade de um programa nesse formato, inserindo-o nos diferentes contextos comunitários, cada um com seus costumes e hábitos, não é tarefa fácil, pois exige dos profissionais olhares voltados para os diferentes grupos sociais considerando suas condições de vida e necessidades coletivas e/ou individuais.

É importante que o profissional atuante na ESF desenvolva habilidades para enfrentar a complexidade das situações sócio territoriais com que irá se deparar. Isso exige dos profissionais responsabilidades e, sobretudo, rompimento dos muros que cercam os serviços de saúde, em favor de uma aproximação gradual entre implementadores e beneficiários. É preciso que o profisssional não só exerça sua função utilizando os recursos empregados na atenção básica para a prevenção e promoção da saúde, mas também se preocupe em ampliar seu conhecimento e compreensão a respeito do complexo processo saúde-doença e dos contextos socioculturais e territoriais que o envolvem.

\section{Atribuições da equipe de profissionais da ESF}

A composição da equipe de profissionais da ESF depende de critérios, como capacidade instalada da unidade; quantidade de pessoas que a equipe terá que assistir; análise dos determinantes do processo de saúde; número de pessoas acometidas por doenças; integralidade da atenção; possibilidades e extensão da área (BRASIL, 2012).

Recomenda-se, no entanto, que a equipe seja composta, no mínimo, por um médico, um enfermeiro, dois auxiliares ou técnicos de enfermagem, seis agentes comunitários, um dentista e um auxiliar de consultório dentário. Outros profissionais podem fazer parte dessa equipe, Barbarói, Santa Cruz do Sul, n. 56, p.<141-163>,jan./jun. 2020 
como psicólogos, assistentes sociais e outros. Embora algumas atividades sejam comuns a todos os profissionais, existem atribuições específicas a cada categoria profissional.

O Ministério da Saúde aconselha que os profissionais residam no município onde atuam. Quanto aos ACS, considera-se que eles devem residir próximos das localidades onde a ESF está implantada, para que seja possível elevar o vínculo com as famílias e com a comunidade local, componentes estruturadores da identidade cultural.

A equipe que atua na ESF deve participar do processo de territorialização e mapeamento para identificar sua área de atuação, por meio de visitas domiciliares e/ou a ambientes coletivos, buscando identificar os diferentes grupos, famílias e indivíduos que possam estar expostos a riscos e vulnerabilidades. Orienta-se que as equipes devem trabalhar unidas, de maneira cooperativa e procurar atender, ao máximo, as necessidades da população sob sua responsabilidade.

Os ACS devem estabelecer vínculos entre a equipe e a população adstrita à UBS; realizar atividades e ações educativas com as famílias visando à promoção da saúde; cadastrar e manter o cadastro atualizado da população da micro área onde atua; fazer prevenção das doenças e dos agravos; identificar situações de riscos; realizar controle de doenças e epidemias, por meio de campanhas; realizar ações isoladas e coletivas em vista de atender às necessidades da população pela qual é responsável.

Para que o ACS exerça suas atividades, algumas competências e habilidades devem se desenvolver: conhecer o território, os problemas da comunidade e suas potencialidades de se desenvolver social e economicamente (BRASIL, 2012).

\section{Perfil da implantação da ESF no Brasil}

Ao analisar a ESF, desde os primeiros momentos de sua implantação como PSF, constata-se que o Brasil experimentou crescente aumento em relação ao número de equipes em território nacional. Isso se deve às necessidades da população, ao porte populacional dos municípios e ao apoio do governo. A ESF é considerada ação prioritária de reorganização da AB de saúde, é a porta de entrada para o sistema de saúde, o SUS (BRASIL, 1997).

Os dados numéricos apresentados a seguir revelam a necessidade e importância da ESF, e representam a situação inicial e atual da implantação da ESF no Brasil, para os meses de dezembro dos anos de 1998, 2001 e 2018. Embora os primeiros movimentos para implantação da ESF tenham se iniciado em meados de 1994, destaca-se que os dados apresentados, para o total do país e segundo as suas regiões, foram divulgados no MS e DAB somente a partir de 1998

Barbarói, Santa Cruz do Sul, n. 56, p.<141-163>,jan./jun. 2020 
Em relação à proporção de ACS implantados no Ministério da Saúde (MS), os dados revelam um aumento expressivo de profissionais no país, no período de 20 anos, perfazendo mais de 233\%, passando-se de 78.705 agentes em 1998 a 262.325 ACS implantados em 2018. Quanto às Equipes de Saúde da Família (ESF), observa-se que, de 1998 a 2018, o aumento do número de municípios com ESF é de 4341, alcançando em 2018, 5.475 municípios com equipes. Considerando as equipes implantadas no país, de 3.062 equipes em 1998, alcançou-se o total de 42.975 equipes em 2018, evidenciando-se o aumento de 14 vezes mais equipes cadastradas. No que se refere às equipes de saúde bucal credenciadas pelo MS, observa-se que, no ano de 1998, não havia nenhuma equipe credenciada. Os dados apenas aparecem no sistema a partir de 2001, perfazendo o total de 3.071 equipes enquanto, em 2018, o total se eleva para 25.914 equipes.

Quadro 1: Credenciamento e implantação das estratégias de Agentes Comunitários de Saúde, Saúde da Família e Saúde Bucal no Brasil (dezembro dos anos de 1998, 2001 e 2018).

\begin{tabular}{|c|c|c|c|c|c|c|c|c|c|c|}
\hline \multirow[b]{2}{*}{ Ano } & \multirow[b]{2}{*}{ Mês } & \multirow[b]{2}{*}{ População } & \multicolumn{3}{|c|}{ Agentes Comunitário de Saúde } & \multicolumn{4}{|c|}{ Equipes de saúde da Família } & \multirow{2}{*}{$\begin{array}{c}\begin{array}{c}\text { Equipes da } \\
\text { saúde } \\
\text { bucal }\end{array} \\
\text { Credenc. } \\
\text { M. S. } \\
\end{array}$} \\
\hline & & & $\begin{array}{c}\text { Credenc } \\
\text { M. S. }\end{array}$ & $\begin{array}{l}\text { Cadast } \\
\text { Sistema } \\
\end{array}$ & Implant. & $\begin{array}{c}\text { Munic } \\
\text { com ESF } \\
\end{array}$ & $\begin{array}{c}\text { Credenc. } \\
\text { M. S. }\end{array}$ & $\begin{array}{l}\text { Cadast. } \\
\text { Sistema }\end{array}$ & Implant. & \\
\hline 1998 & 12 & 159.644 .929 & 86.231 & 79.677 & 78.705 & 1.134 & 3.353 & 3.083 & 3.062 & 0 \\
\hline 2001 & 12 & 172.385 .826 & 184.449 & 155.783 & 152.865 & 3.682 & 19.253 & 13.318 & 13.155 & 3.071 \\
\hline 2018 & 12 & 206.114 .067 & 276.791 & 271.431 & 262.325 & 5.475 & 45.417 & 43.950 & 42.975 & 25.914 \\
\hline
\end{tabular}

Fontes: Ministério da Saúde. Departamento de Atenção Básica. MS/SAS/DAB e IBGE. (BRASIL, 2019).

No que se refere aos profissionais cadastrados no Ministério da Saúde por regiões do país observa-se que, tanto em 1998 quanto em 2018, a região Nordeste registra os maiores totais de ACSs e equipes da ESF implantados, apresentando um crescimento percentual de $115,7 \%$ no período de 20 anos até 2018. No mesmo período, o número de equipes da ESF implantadas aumentou 12,7 vezes, totalizando 15.646 equipes. Quanto às equipes de saúde bucal credenciadas pelo Ministério, a região nordestina foi novamente a de maior destaque, com 12.174 equipes credenciadas em 2018.

No que se refere aos ACS implantados, conforme cálculo a partir dos dados do Ministério (Quadro 2), no período de 20 anos até 2018, o aumento do número de agentes no Sudeste, em 10,8 vezes a mais, seguido pela região Sul, com 4,9 vezes agentes a mais, e pelo Norte (2,9 vezes a mais) e Centro Oeste (2,6 vezes a mais). Já no caso das equipes da ESF Barbarói, Santa Cruz do Sul, n. 56, p.<141-163>, jan./jun. 2020 
implantadas, no mesmo período, a região Norte se destaca com um acréscimo de equipes da ordem de 21,3 vezes a mais, seguida pelas regiões Sul $(17,6)$, Sudeste $(14,3)$ e Centro Oeste com um aumento de 10,1 vezes a mais de equipes implantadas até 2018.

No tocante à saúde bucal, nota-se que, em 1998, não houve implantação de serviços em nenhuma das regiões e, somente a partir do ano de 2001, os dados passam a ser registrados. A Tabela 2 evidencia, para 2018, que a região Nordeste também foi a que mais se destacou, com 12.174 equipes credenciadas, seguidas pelo Sudeste, o Sul, o Centro-Oeste e a região Norte, com apenas 2.196 equipes credenciadas.

Além disso, é importante chamar atenção ao fato de que as regiões Nordeste, Norte e Centro-oeste são as que apresentam a mais alta proporção de ACS por 1.000 habitantes, nas duas datas consideradas. No caso dos ACS, em 2018, essa razão foi, respectivamente às regiões citadas, 1,8 ACS/1.000 habitantes, 1,7 e 1,1. Quanto às ESF, essa razão foi, em 2018, menos expressiva que a razão de ACS por 1.000 habitantes, e em ordem decrescente, mais alta nas regiões Nordeste $(0,27)$ ESF/1.000 habitantes), Centro-oeste $(0,21)$ e Sul do país $(0,21)$.

Para o Brasil como um todo, em 2018, existiam 1,27 ACS/1.000 habitantes e 0,21 ESF/1.000 habitantes. Com esse cenário, constata-se que a tendência crescente de equipes e de agentes implantados se mostra relativamente mais importante no Nordeste, convergindo com a orientação do governo federal às políticas sociais, mas ainda aparentemente insuficiente ao considerar a proporção de agentes e equipes por 1.000 habitantes.

Quadro 2: Teto, credenciamento e implantação das estratégias de Agentes Comunitários de Saúde, Saúde da Família e Saúde Bucal das regiões geográficas do Brasil (dezembro de 1998 e 2018).

\begin{tabular}{|c|c|c|c|c|}
\hline \multirow[b]{2}{*}{ Região } & \multirow[b]{2}{*}{ População } & $\begin{array}{c}\text { Agentes Comunitário de } \\
\text { Saúde }\end{array}$ & Equipe de saúde da Família & \multirow{2}{*}{$\begin{array}{c}\text { Equipe da Saúde } \\
\text { bucal }\end{array}$} \\
\hline & & Implantados & Implantados & \\
\hline \multicolumn{5}{|c|}{ Ano de 1998} \\
\hline Centro Oeste & 10.769 .249 & 7.172 & 318 & 0 \\
\hline Nordeste & 45.334. 385 & 47.381 & 1.230 & 0 \\
\hline Norte & 11.612 .674 & 10.402 & 165 & 0 \\
\hline Sudeste & 68.065 .957 & 7.324 & 996 & 0 \\
\hline Sul & 23.862 .664 & 6.426 & 353 & 0 \\
\hline \multicolumn{5}{|c|}{ Ano de 2018} \\
\hline Centro Oeste & 15.660 .998 & 18.683 & 3.235 & 2.258 \\
\hline Nordeste & 56.915 .936 & 102.207 & 15.646 & 12.174 \\
\hline Norte & 17.740 .418 & 30.364 & 3.528 & 2.196 \\
\hline
\end{tabular}

Barbarói, Santa Cruz do Sul, n. 56, p.<141-163>,jan./jun. 2020 


\begin{tabular}{|c|c|c|c|c|}
\cline { 3 - 5 } Sudeste & 86.356 .952 & 79.380 & 14.336 & 6.286 \\
\hline Sul & 29.439 .773 & 31.691 & 6.230 & 3.000 \\
\hline
\end{tabular}

Fontes: Ministério da Saúde. Departamento de Atenção Básica. MS/SAS/DAB e IBGE (BRASIL, 2019).

Os dados anteriormente apresentados quanto à implantação das equipes de ESF vão ao encontro dos resultados de estudo realizado por Alves et al. (2014), em que os autores afirmam ter observado panorama semelhante em relação à implantação das equipes da ESF no Brasil desde os anos de 2000. Os autores declaram que houve crescimento expressivo em relação à cobertura da população e que tal fenômeno aconteceu em ritmos diferentes conforme as regiões. Isso talvez se deva ao fato de o crescimento populacional dos municípios também ter ocorrido em ritmos diferentes, exigindo um conjunto de ações por parte dos gestores e do reconhecimento da necessidade de mais ESF e mais recursos, para melhor organizar os serviços nos municípios de maior índice populacional e nos mais carentes.

A literatura mostra a preocupação de alguns órgãos de pesquisa com a divulgação de dados que demonstraram a situação epidemiológica e de cobertura pela ESF no Brasil. A Pesquisa Nacional por Amostra de Domicílio (PNAD), realizada em 2008, apontou, na época, maior cobertura na região Nordeste e menor na região Sudeste. Tal diferença foi, também, observada na Pesquisa Nacional de Saúde (PNS). Os estudos apontaram o crescimento populacional na maior parte dos estados e, por conseguinte, um aumento na cobertura populacional. Os dados apresentados por esses órgãos de pesquisa são bem próximos dos dados do Departamento da Atenção Básica (BRASIL, 2008).

O Programa Nacional de Atenção Básica (PNAB) resultou da experiência que se somou ao longo dos anos por meio das práticas e atuação de diferentes atores na tentativa de consolidar o SUS, tais como, os movimentos sociais e de trabalhadores e a participação ativa dos governos federal, estadual e municipal (BRASIL, 2012).

Como forma de acompanhar e avaliar o PNAB realizou-se um estudo denominado “Atenção primária à Saúde em 2016: avanços, desafio e resultados”, cujos eixos analisados de cobertura em território brasileiro foram: os programas Mais Médicos (PMM), Agentes Comunitários de Saúde (PACS), Estratégia de Saúde da Família (ESF) e Núcleos de Apoio à Saúde da Família (NASF) (OBSERVATÓRIO DE ANÁLISE POLÍTICA EM SAÚDE, 2017). Os resultados desse estudo revelam que houve aumento pouco expressivo nos programas ACS, ESF e NASF de 2015 para 2016, visto que a cobertura populacional do PACS em 2015, igual a $66,63 \%$, passou para 66,66\% em novembro de 2016, perfazendo o total de 5.507 equipes. Já em relação às equipes de ESF, constata-se um aumento de aproximadamente $0,2 \%$, ou seja, em 
2015 a cobertura igual a 63,45\% passa, em novembro de 2016, a 63,62\%, com aproximadamente 40.067 equipes já implantadas, atendendo o total de 5.382 municípios em território brasileiro. Quanto aos NASF, também se constata aumento pouco expressivo das equipes, passando de 2.343 para 2.509 em 2016.

Malta et al. (2016) participam deste debate e revelam que a maior parte da população brasileira está coberta por esses serviços. Os autores enfatizam que pode ocorrer a diminuição da iniquidade com o incentivo de implantação da ESF e das ações em saúde dispensadas às famílias e consideram expressiva essa cobertura, mas, para tais autores, outras ações são necessárias para alcançar a resolutividade e qualidade da atenção como forma de garantir atendimento equânime à população.

\section{Processos e instrumentos de implantação da ESF: a dimensão articuladora dos territórios}

Os processos e dinâmicas sócio territoriais tornaram-se desafiadores para o conhecimento e a gestão pública.

O conceito ampliado de saúde deve ser estimulador e reorganizador do sistema de saúde, pois em seu sentido ampliado, a saúde coletiva resulta de diferentes condições além do acesso aos serviços de saúde propriamente ditos: tais como a habitação, renda, alimentação, educação, meio ambiente, transporte, emprego e outros. Esse olhar ampliado sobre a saúde implica no compromisso de se efetivar a implementação das políticas de saúde junto às populações, que estão organizadas socialmente e em famílias, nos espaços de vida com os quais esses indivíduos se relacionam (ARAUJO et al., 2017).

Muito embora a saúde seja considerada um direito fundamental inalienável à população, nos dias atuais ainda se observam fragilidades nas questões referentes à efetivação da oferta dos serviços de saúde e do acesso a esses serviços.

Atendendo ao conceito ampliado de saúde, a apropriação do território pela equipe da ESF permite o reconhecimento das condições de vida das pessoas nos diferentes contextos socioculturais e territórios que ocupam, constroem, trabalham e vivem.

A esse respeito, Araújo et al. (2017) afirmam que a garantia dos direitos à saúde se dá por meio da organização de macro processos tais como: territorialização, cadastramento das famílias, classificação das áreas de riscos, identificação das famílias vulneráveis a riscos e estabelecimento de vínculos entre a equipe implementadora de políticas de saúde. Para os autores, estes elementos são fundamentais para a implementação de estratégias de Atenção Primária da Saúde (APS) e, consequentemente, para a gestão da saúde da população. 
Nessa perspectiva de organização do setor de saúde no modelo de APS propõe-se, então, a elaboração de novas estratégias que permitam maior cobertura dos serviços de saúde da população, de modo que o cuidado seja ofertado para além dos muros da ESF, permitindo a oferta de ações cada vez mais efetivas para a coletividade e, assim, diminuindo a desigualdade no acesso aos serviços.

Warschauer e Carvalho (2014) afirmam que essas ações de reorganização do sistema de saúde são necessárias e devem estimular a criação de mecanismos de participação e mobilização social, de movimentos de trabalhadores da área de saúde e de outros setores e estimular a captação de recursos financeiros para além dos que a saúde possui, colocando à saúde o desafio da intersetorialidade como nova forma de organização do sistema de saúde.

Ao longo da história, após a implementação do SUS, percebe-se que ainda existem dificuldades a caminho da oferta de serviços de saúde de qualidade que, de fato, atendam às necessidades reais da população, principalmente por parte dos municípios menores. A cooperação intersetorial, intermunicipal e interdisciplinar talvez seja uma das estratégias que os municípios de menor porte poderiam priorizar para afirmar sua autonomia, consumar a descentralização da política e garantir os princípios de universalidade, integralidade e equidade propostos pelo SUS.

Diante disso, apresenta-se, a seguir, uma breve contextualização sobre alguns instrumentos articuladores da implementação das políticas públicas de saúde nos territórios: territorialização e cadastro; direito à saúde e cooperação intersetorial, intermunicipal e interdisciplinar.

\section{Territorialização e cadastro da população}

Os primeiros passos em relação à implantação do PSF se deram no ano de 1991, com a criação do PACS, considerado um momento de transição para a implantação do PSF que, em 2006, passou a ser chamado de ESF.

As diretrizes da ESF foram estabelecidas pelo Ministério da Saúde (MS) desde 1997, com orientação para sua implantação. A Estratégia foi implantada com a finalidade de reorganizar as práticas de trabalho; capacitação dos profissionais por meio de educação continuada; estabelecer equipes multiprofissionais; fazer diagnóstico da saúde da comunidade; planejamento de ações para população local; estimular a ação intersetorial; acompanhar, avaliar e realizar o controle social.

A ESF representa a primeira proposta de contato entre profissionais e população por meio dos serviços municipais de saúde. Ela deve garantir assistência total a toda a população, 
desde o acolhimento à referência (encaminhamento dos pacientes aos serviços especializados) e contra referência (a devolução dos pacientes para unidade de origem). Os estudos prévios para a implantação da ESF exigem que seja analisada a adscrição da população, seu cadastramento, composição e atribuições das equipes (BRASIL, 1997). Além disso, busca-se reorganizar os olhares dos gestores, implementadores e usuários, uma vez que todos estão envolvidos na gestão da Estratégia.

$\mathrm{Na}$ área da saúde, o território é entendido como uma rede de serviços que se estrutura em regiões e municípios e pode possibilitar a efetivação do direito à saúde. Por isso, é importante que o território e seu entorno sejam bem estudados antes da implantação da ESF.

Essa prática é denominada de "territorialização", e seu propósito é reconhecer as condições físicas e sócio territoriais das áreas de atuação. O Mapeamento, instrumento correlato à territorialização, consiste na identificação de equipamentos e condições favoráveis e/ou desfavoráveis para a população habitante (escolas, igrejas, áreas com esgoto a céu aberto, pontos de drogas) (FARIA, 2013).

Essas práticas garantem a qualidade dos serviços que serão ofertados, pois, por meio delas, o profissional pode conhecer melhor a população que será por ele cuidada, o que facilita o direcionamento de ações e assistência mais assertivas.

Para garantir a qualidade de suas ações, a ESF deve definir territórios de sua atuação, pelos quais os agentes de saúde (ACS) se responsabilizarão. A cada equipe e agentes cabe a responsabilidade por uma área e pelas famílias que ali residem.

A Política Nacional de Atenção Básica estabelece que cada equipe da ESF deve ser responsável por, no máximo, 4.000 pessoas, sendo a média recomendada de 3.000 por estratégia. Em casos de populações mais vulneráveis, esse número deve ser reduzido, em média para 2.000 pessoas por equipe, evidenciando que a estratégia deve ser flexível (BRASIL, 2012).

A adscrição de usuários, outra ferramenta utilizada na territorialização, pode ser definida como processo de inscrição, cadastro, apresentação e criação de vínculos entre pessoas, famílias, comunidades e as equipes de profissionais, para reafirmá-los como referências para o cuidado da saúde. Esse processo contribui para a construção de relações de afetividade e confiança entre o usuário e o profissional da saúde.

Essa distribuição geográfica para a prestação de serviços diz respeito, então, aos territórios adstritos, lugar operacional para o qual as ações e os serviços das equipes de saúde são organizados, para a promoção e prevenção da saúde da população.

Em termos práticos, a territorialização subentende o processo de aproximação ao território adscrito com um olhar mais abrangente e aprofundado, procurando-se identificar e Barbarói, Santa Cruz do Sul, n. 56, p.<141-163>,jan./jun. 2020 
analisar a localização geográfica e as características sociodemográficas, econômicas e culturais dos territórios; as condições de moradia da população cadastrada; as condições ambientais e de vulnerabilidade; a disponibilidade de equipamentos públicos e sociais; dados epidemiológicos da população e de doenças crônicas etc (FARIA, 2013).

Silva Junior et al. (2010) ressaltam que a territorialização é a base do trabalho das Equipes da ESF, cuja principal finalidade é eleger prioridades para resolução de possíveis problemas identificados nos territórios de atuação. Essa prática estimula o estabelecimento de vínculos e, por conseguinte, reflete positivamente sobre a acessibilidade da população aos serviços de saúde. Não se trata de uma simples técnica de coleta de dados de um dado território, mas, sim, da forma como este será interpretado e atualizado, periodicamente, pelas equipes com reflexos, portanto, na redefinição de estratégias e ações mais adequadas (BRASIL, 2008).

$\mathrm{Na}$ literatura consultada, foram encontrados diferentes termos que representam a prática da divisão territorial realizada pela equipe da ESF, para orientar e operacionalizar ações em saúde: mapeamento, áreas adstritas, áreas de abrangência de unidades de saúde, microáreas e outros. Independente da nomenclatura, o mapeamento dos territórios constitui um instrumento fundamental para o planejamento detalhado das ações em saúde e sua gestão (MÔRA et al., 2013).

$\mathrm{Na}$ ESF, mapear o território significa delimitar as microáreas (nos municípios) de atuação dos profissionais. Os profissionais da ESF elaboram mapas inteligentes que possibilitam melhor visualização para a equipe das situações diferenciadas das populações adstritas. O mapeamento das microáreas é realimentado por ações de territorialização e indicadores diversos, contribuindo, assim, para a realização do diagnóstico da comunidade e para a melhoria da qualidade da assistência ofertada.

Para elaboração do mapeamento, as equipes devem ir a campo para levantamento de informações. Após essa fase, os profissionais elaboram um mapa, utilizando cores diferentes previamente definidas pelo Ministério da Saúde com a finalidade de destacar o perfil e a vulnerabilidade das condições de saúde da população adstrita, indicados pelas doenças crônicas, a existência de hipertensos, diabéticos, acamados, alcoólicos, epiléticos, pacientes com necessidades especiais, obesos, gestantes etc. (CARINE et al., 2011).

A etapa posterior consiste, na identificação de pontos favoráveis (acesso, pontos de táxi, ônibus, farmácias, laboratórios, equipamentos culturais e de lazer) e na identificação das microáreas de risco, pela probabilidade de alagamentos ou desmoronamentos.

O mapa inteligente qualifica os problemas e as questões passíveis de intervenções pela equipe, organiza o conhecimento dos territórios e facilita a visualização e comunicação das Barbarói, Santa Cruz do Sul, n. 56, p.<141-163>,jan./jun. 2020 
equipes da ESF no território. Para de fato funcionar como ferramenta de diagnóstico local, é importante que os profissionais, atuantes em diferentes territórios, considerem o mapa como elemento dinâmico em função da necessidade de permanente atualização.

Uma das principais características da ESF é promover atenção continuada por meio da equipe multiprofissional habilitada. Por isso, a prática do trabalho em conjunto, colaborativo, com envolvimento de todos os membros da equipe é fundamental. É importante salientar que existem atividades comuns para toda a equipe e, também, atividades específicas de cada área a que pertencem os profissionais, sendo responsabilidade de cada um apenas as atividades legais previstas para o exercício de sua profissão (BRASIL, 2011).

Integrado à territorialização e ao mapeamento, o cadastro das famílias e pessoas é outro instrumento importante na implementação e avaliação da política e dos serviços. Ele é feito por meio de visitas domiciliares realizadas pelos profissionais, respeitando o preestabelecido na fase da adscrição do território, e possibilitando a identificação das condições familiares. Nessa fase, vínculos iniciais são estabelecidos entre os profissionais da saúde e a comunidade; os profissionais têm a oportunidade de conhecer a população sob sua responsabilidade e informar sobre os serviços que irão prestar, além de horários, datas, locais da unidade da saúde que deverão ser a referência inicial para a população.

O cadastro é uma ferramenta importante para a modalidade de atenção à saúde, pois, a partir dele, define-se a população que será vinculada a cada equipe da ESF. A população cadastrada não é fornecida por nenhuma fonte oficial de dados, mas pela população que efetivamente reside no território. De fato, o bom uso dessa ferramenta pode melhorar, em muito, a gestão, a programação de ações e a oferta dos serviços necessários às populações (BRASIL, 2012).

A partir daí, os profissionais elaboram prontuários para cada família, contendo dados importantes que podem ser utilizados em prol da comunidade, no momento da classificação das famílias, nas etapas de planejamento e implantação das ações a serem desenvolvidas naquele território, com direcionamento específico para diferentes perfis demográficos (jovens, idosos, gestantes etc.) e da saúde coletiva.

\section{Direito à saúde e cooperação intersetorial, intermunicipal e interdisciplinar}

A CF de 1988 garantiu à sociedade diversos direitos, dentre eles, destacam-se a Seguridade Social e o direito à Saúde. Dentre os diversos capítulos dispostos na CF, ressaltamse os artigos 196 e 198, que definem o arcabouço de sustentação ao SUS, garantindo a saúde a todos como direito, por meio de acesso universal igualitário para toda a sociedade no que diz 
respeito à oferta de ações de promoção, proteção e recuperação da saúde e, sobretudo, atendimento integral à população (BRASIL, 1988).

Analisando a saúde pela lógica social, Dallari (1988) afirma que:

\begin{abstract}
o direito à saúde privilegia a igualdade. As limitações aos comportamentos humanos são postas exatamente para que todos possam usufruir igualmente as vantagens da vida em sociedade. Assim, para preservar-se a saúde de todos é necessário que ninguém possa impedir outrem de procurar seu bem-estar ou induzi-lo a adoecer. Essa é a razão das normas jurídicas que obrigam à vacinação, à notificação, ao tratamento, e mesmo ao isolamento de certas doenças, à destruição de alimentos deteriorados e, também, ao controle do meio ambiente, das condições de trabalho (DALLARI, 1988, p. 59).
\end{abstract}

Assim, a saúde faz parte do conjunto de direitos sociais e é considerado direito fundamental do cidadão, cuja finalidade consiste em diminuir a desigualdade entre as populações.

O homem é um ser bio-psico-social com diferentes necessidades, mas, pensar no bem comum dos homens é pensar coletivamente, uma vez que uma única política não é capaz de garantir e sanar suas necessidades sociais. O Estado é responsável por garantir a constituição de uma sociedade diversificada, com olhares diferenciados para as diversas necessidades de provisão: educação, trabalho, habitação, saúde, segurança, dentre outros, pois a vida é tecida por um conjunto de fatores propondo articulação entre variados saberes e ciências (INOJOSA, 2001).

O conceito de saúde é complexo e, para compreendê-lo, torna-se necessário construir uma visão ampliada, sendo indispensável que os atores envolvidos nesse processo busquem conhecimentos em outras teorias que ultrapassem as políticas sociais. Apesar da CF de 1988 ser pensada para atender às necessidades da população, e embora o texto constitucional comtemple o desenho intersetorial, as práticas intersetoriais ainda se configuram como processos inovadores e, em sua maioria, ainda pouco viabilizadas.

A esse respeito, Inojosa (2001) faz a seguinte consideração sobre o conceito de intersetorialidade:

a articulação de saberes e experiências com vistas ao planejamento, para a realização e a avaliação de políticas, programas e projetos, com o objetivo de alcançar resultados sinérgicos em situações complexas. Trata-se, portanto, de buscar alcançar resultados integrados visando a um efeito sinérgico. Transpondo a ideia de transdisciplinaridade para o campo das organizações, o que se quer, muito mais do que juntar setores, é criar uma nova dinâmica para o aparato governamental, com base territorial e populacional (INOJOSA, 2001, p. 105).

O Brasil possui uma vasta dimensão continental com características próprias e diversificadas em termos culturais, étnicos, sociais, econômicos, ambientais, de complexidades 
variadas, o que é desafiador para que os gestores e implementadores de serviços públicos de difícil acesso às populações sejam, de fato, resolutivos, eficientes e com ações humanizadas.

Nesse sentido, Silva et al. (2017) afirmam que os municípios brasileiros, sobretudo os de pequeno porte, enfrentam dificuldades para oferecer serviços de saúde de qualidade à população sobre sua responsabilidade. Com o processo de descentralização proposto pelo SUS, essas dificuldades se agravaram, emergindo a necessidade de elaboração de estratégias de produção para efetivação das políticas públicas, tais como a cooperação intermunicipal ou de “cooperativismo horizontal".

A cooperação intermunicipal demonstra ser importante ferramenta para efetivação das ações de saúde nos municípios, pois é capaz de gerar estratégias locais regionalizadas reduzindo possíveis fraquezas dos governos municipais, fortalecendo sua ação conjunta para atingir objetivos comuns (ABRUCIO, 2017).

As ações de saúde devem ser compreendidas e vislumbradas pela lógica da complexidade e, portanto, a integração de outras áreas e ciências se torna necessária. O processo interativo e a intensidade das trocas entre as diferentes ciências produzem forças e estratégias para transpor barreiras em termos de resolutividade.

A complexidade do conceito de saúde exige a adoção de novas posturas em relação à produção do conhecimento. O campo da saúde, em especial a área da saúde pública, é demarcada pela correlação de forças das diferentes disciplinas que tentam se articular, sem perder de foco a consciência social e política.

A esse respeito, Scherer e Pires (2011) afirmam que a busca pela resolução de problemas que inquietam a humanidade abre caminho para a interdisciplinaridade, considerada pelas autoras peça-chave para a resolutividade de problemas, pois a utilização dos conhecimentos de diferentes ciências é indispensável para a compreensão dos fenômenos que acontecem no mundo e a intervenção sobre os mesmos.

Assim, vale-se hoje da interdisciplinaridade como meio de aproximar, comparar, relacionar e integrar os conhecimentos e promover uma filosofia de trabalho coerente com as diversas problemáticas interligadas e demandas atuais. A interdisciplinaridade se caracteriza pela capacidade de troca de conhecimentos entre as pessoas e pela incorporação de diferentes disciplinas, "propõe um avanço em relação ao ensino que denominamos tradicional, como mera transmissão e recepção de conhecimentos sem que estes tenham significados na formação" é o que afirma Oliveira (2016, p. 191). A integração das áreas de conhecimento enriquece e transforma os conceitos, possibilitando rever a práxis e a busca de resolução de problemas 
concretos, minimiza a fragmentação e a descontextualização do conhecimento (GOMES, DESLANDES, 1994).

Considera-se que, no âmbito do poder público ou do conhecimento, as ações setoriais não sejam dissociadas, pois as iniciativas de associação entre as diferentes políticas sociais e ciências geram e fortalecem os recursos que serão implementados para pensar e resolver os problemas da sociedade nos diferentes territórios. Assim, pode-se promover melhorias às condições de vida da população, possibilitar o acompanhamento das necessidades e a implementação de ações integradas, mais condizentes com os anseios da sociedade, além de garantir a integralidade da atenção e do cuidado, melhorar o acesso da população e, talvez, assim, obter resultados efetivos no sentido da redução de desigualdades entre os povos.

\section{Considerações finais}

O estudo procurou retratar os processos de reorganização da política de saúde no país que conduziu à implantação do SUS. Nesse contexto, chegou-se à ESF, que é um modelo de atenção que segue os princípios básicos do SUS para a redução da iniquidade: promoção da universalização, equidade, integralidade e participação da comunidade. Vimos as atribuições, importância e o aumento expressivo das equipes de ACS e da ESF em território brasileiro, trazendo melhoras na cobertura da assistência à população, com destaque para a região Nordeste.

Outro elemento importante destacado no texto refere-se à necessária prática assistencial multi/interdisciplinar. Essa complementaridade entre as diferentes disciplinas e os distintos profissionais pode auxiliar na compreensão, no planejamento, na gestão e organização das ações a serem dispensadas às diferentes populações em seus territórios de vida. Além disso, destacase a importância de os profissionais poderem reorganizar sua visão dos problemas estritos ao campo da saúde, alargando-a e aprofundando-a a partir do conceito ampliado de saúde, ao contemplar as condições do lugar de vida social.

A territorialização da política de saúde foi, por isso, identificada como importante ferramenta para o conhecimento das populações beneficiárias dos serviços e dos territórios em que vivem, e é fundamental para o planejamento e a gestão das ações das unidades de ESF e o processo continuado de avaliação da política.

A territorialização e os mapeamentos são fundamentais para a construção de laços de confiança entre profissionais e beneficiários e a avaliação de implicações das condições sociais e territoriais para a saúde das populações. Essas ações possibilitam o processo de vigilância e atenção à saúde e a constante reorganização do processo de atuação profissional e, ainda, podem 
contribuir na melhoria do lugar de vida das pessoas ao identificarem necessidades de transformação dos territórios em favor de uma saúde de alcance integral, do indivíduo ao ambiente social do lugar em que vivem.

Assim sendo, chega-se à seguinte inferência: o território, a saúde das populações e as políticas de saúde pública são elementos interligados que contribuem para a redemocratização da sociedade, pois contêm as possibilidades de seu fortalecimento, uma vez que interferem nas diretrizes e decisões que possam garantir o exercício da cidadania às populações em todo o país. Aqui reside o grande potencial de aprimoramento das análises e da própria política de saúde, no caso, considerando a ESF particularmente.

A distribuição e qualificação das unidades (em seus diferentes níveis de atenção) e das equipes e o aprimoramento constante do contato entre profissionais e as populações em seus territórios de vida e contextos socioculturais constituem a dimensão inovadora da política de saúde em sua condição de verdadeira política de caráter social e territorial. Assim sendo, entreabre-se um espaço para a integração de políticas e ações, no âmbito municipal e, mesmo, no regional, e à interlocução de campos do conhecimento que só tendem a contribuir para o direito à saúde e ao lugar de vida saudável.

\title{
FAMILY HEALTH STRATEGY (FHS) IN BRAZIL: THE ARTICULATING CONDITION OF TERRITORY
}

\begin{abstract}
This is an, descriptive and analytical study, which aims to examine the process of implementation of the Family Health Strategy (FHS) in Brazil providing public health services in the context of the Sistema Unico de Saúde (SUS). For this, the literature and data review of the Ministry of Health was used. In this context, the value of multi and interdisciplinary care practice and the territorializing and mapping are important tools for the constant evaluation of the program as well as the planning of intersectoral actions and the management of the units and teams of the FHS, because it is possible to know the population to be served and their socioterritorial and health conditions. In 20 years there has been an increase in Community Health Agents (ACS) and FHS teams in all regions of the country, but the reorganization of Primary Health Care $(\mathrm{AB})$ remains one of the biggest challenges to improve management and the provision of services, and therefore for the teams of professionals involved in it.
\end{abstract}

Keywords: Community Health Agent; Territorializing; Collective health.

\section{ESTRATEGIA DE LA SALUD DE LA FAMILIA: LA DIMENSIÓN ARTICULADORA DEL TERRITORIO}

\section{Resumen}

Este es un estudio descriptivo y analítico, y tiene por objeto examinar el proceso de implantación de la Estratégia de la Salud de la Familia (ESF) en Brasil efectivizando servicios de salud pública en el contexto del Sistema Único de Salud (SUS). Para eso, ha utilizado la revisión de bibliografía y datos del Ministerio de la Salud. En este sentido, sobresale el valor Barbarói, Santa Cruz do Sul, n. 56, p.<141-163>,jan./jun. 2020 
de la práctica asistencial multi e interdisciplinar y de la territorialización y mapeo como importantes herramientas para permanente evaluación del plan, la planificación de las acciones intersectoriales y la gestión de las unidades y equipos de la ESF, pues permiten el conocimiento de las poblaciones que deben ser atendidas y de las condiciones socio territoriales y de salud de las mismas. Se observa en 20 años un crecimiento de Agentes Comunitarios de Salud (ACS) y de los equipos de la ESF en todas las regiones del país, pero la reorganización de la Atención Básica $(\mathrm{AB})$ en salud sigue siendo uno de los mayores retos para el perfeccionamiento de la gestión y suministro de los servicios, y por lo tanto, para los equipos de profesionales involucrados.

Palabras-llave: Agente Comunitario de Salud; Territorialización; Salud Coletiva.

\section{REFERÊNCIAS}

ABRUCIO, F. L. Cooperação Intermunicipal: experiências de arranjos de desenvolvimento no Brasil. Curitiba: Positivo, 2017.

ALVES, M. G. M. et al. Fatores condicionantes para o acesso às equipes da Estratégia Saúde da Família no Brasil. Saúde Debate. v. 38 (n. esp.) 34-51, 2014. Disponível em: <http://www.scielo.br/pdf/sdeb/v38nspe/0103-1104-sdeb-38-spe-0034.pdf>. Acesso em: 12 de março de 2019.

ARAÚJO, G. B. et al. Territorialização em saúde como instrumento de formação para estudantes de medicina: relato de experiência. Revista SANARE Sobral. v. 16, n. 01, p. 124129, 2017. Disponível em: 〈https://sanare.emnuvens.com.br/sanare/article/view/1103/614>. Acesso em: 10 de dezembro de 2019.

BRASIL. Constituição da República Federativa do Brasil de 1988. Disponível em: <https://www2.senado.leg.br/bdsf/bitstream/handle/id/518231/CF88_Livro_EC91_2016.pdf>. Acesso em: 10 de dezembro de 2019.

Lei n. 8080, de 19 de setembro de 1990. Dispõe sobre as condições para a promoção, proteção e recuperação da saúde, a organização e o funcionamento dos serviços correspondentes e dá outras providências. Diário Oficial da União, Brasília, 20 de setembro de 1990. Disponível em: <http://www.planalto.gov.br/ccivil_03/leis/L8080.htm>. Acesso em: 26 de março de 2019.

Saúde da Família: uma estratégia para a reorientação do modelo assistencial. Coordenação de Saúde da Comunidade. Brasília: Ministério da Saúde, 1997. Disponível em:< http://bvsms.saude.gov.br/bvs/publicacoes/cd09_16.pdf >. Acesso em: 03 de março de 2019.

Vigilância em Saúde: dengue, esquistossomose, hanseníase, malária, tracoma e tuberculose. (Série A. Normas e Manuais Técnicos) (Cadernos de Atenção Básica, n. 21) Ministério da Saúde. Secretaria de Atenção à Saúde. Departamento de Atenção Básica. 2. ed. Brasília: Ministério da Saúde, 2008. Disponível em:

<http://bvsms.saude.gov.br/bvs/publicacoes/cab_n21_vigilancia_saude_2ed_p1.pdf>. Acesso em: 15 de março de 2019.

Portaria $\mathbf{n}^{\mathbf{0}}$ 2.488, de 21 de outubro de 2011. Aprova a Política Nacional de Atenção Básica, estabelecendo a revisão de diretrizes e normas para a organização da Atenção 
Básica, para a Estratégia Saúde da Família (ESF) e o Programa de Agentes Comunitários de Saúde (PACS). Diário Oficial da República Federativa do Brasil, Brasília, n.204, p.55, 24 out. 2011. Disponível em:

<http://bvsms.saude.gov.br/bvs/saudelegis/gm/2011/prt2488_21_10_2011.html>. Acesso em: 03 de abril de 2019.

Política Nacional de Atenção Básica. Secretaria de Atenção à Saúde.

Departamento de Atenção Básica. Brasília: Ministério da Saúde, 2012. Disponível em: <http://189.28.128.100/dab/docs/publicacoes/geral/pnab.pdf>. Acesso em: 03 de março de 2019.

\section{MINISTÉRIO DA SAÚDE. E - GESTOR ATENÇÃO BÁSICA: Informação e}

Gestão da Atenção Básica. Disponível em:

$<$ https://egestorab.saude.gov.br/paginas/acessoPublico/relatorios/relHistoricoCobertura.xhtml >. Acesso em: 13 de outubro de 2019.

CARINE, G. C. et al. Mapa inteligente: planejamento em ações de saúde no município de Santa Cruz do Sul. 2011. Disponível em:

<http://online.unisc.br/acadnet/anais/index.php/salao_ensino_extensao/article/view/10079>. Acesso em: 08 de abril de 2019.

CONSELHO NACIONAL DE SECRETÁRIOS DE SAÚDE - CONASS. Atenção Primária e promoção da saúde. Brasília: CONASS, 2011. Disponível em:

<http://www.conass.org.br/bibliotecav3/pdfs/colecao2011/livro_3.pdf>. Acesso em: 10 de março de 2019.

DALLARI, S. G. O direito à saúde. Revista Saúde Pública. v. 22, n. 1, p, 57 - 63. Fevereiro de 1988. Disponível em:

<http://www.scielo.br/scielo.php?script=sci_arttext\&pid=S0034-89101988000100008>.

Acesso em: 12 de dezembro de 2019.

DALPIAZ, A. K.; STEDILE, N. L. R. Estratégia de Saúde da Família: reflexão sobre algumas de suas premissas. 2011. Jornada Nacional de Políticas Públicas. Disponível em: <http://www.joinpp.ufma.br/jornadas/joinpp2011/CdVjornada/JORNADA_EIXO_2011/IMP ASSES_E_DESAFIOS_DAS_POLITICAS_DA_SEGURIDADE_SOCIAL/ESTRATEGIA_ SAUDE_DA_FAMILIA_REFLEXAO_SOBRE_ALGUMAS_DE_SUAS_PREMISSAS.pdf >. Acesso em: 30 de março de 2019, p. 3 .

FARIA, C. A. P. Ideias, conhecimento e políticas públicas: um inventário sucinto das principais vertentes analíticas recentes. Revista Brasileira de Ciências Sociais. v. 18, n. 51, fevereiro de 2003.

FARIA, H. P. et al. Modelo assistencial e atenção básica à saúde. 2. ed. Belo Horizonte: NESCON/UFMG, 2010. Disponível em:

<https://www.nescon.medicina.ufmg.br/biblioteca/imagem/1792.pdf>. Acesso em: 28 de abril de 2019.

FARIA, R. M. A territorialização da atenção primária à saúde no Sistema Único de Saúde e a construção de uma perspectiva de adequação dos serviços aos perfis do território. Revista Brasileira de Geografia. Hygeia, v. 9, n. 16, p. 131 - 147, junho de 2013. Disponível em: 
<http://www.seer.ufu.br/index.php/hygeia/article/view/19501/12458>. Acesso em: 20 de abril de 2019.

GOMES, R.; DESLANDES, S. F. Interdisciplinaridade na Saúde Pública: um campo em construção. Revista Latino-Americana Enfermagem. v. 2, n. 2, p. 103 - 114, julho de 1994. Disponível em: <http://www.scielo.br/pdf/rlae/v2n2/v2n2a08.pdf>. Acesso em: 13 de dezembro de 2019.

INSTITUTO BRASILEIRO DE GEOGRAFIA E ESTATÍSTICA - IBGE. Pesquisa nacional por amostra de domicílios; um panorama da saúde no Brasil; acesso e utilização de serviços, condições de saúde e fatores de risco e proteção à saúde 2008,2010 . Rio de Janeiro: IBGE, 256 p. Disponível em:

<http://bvsms.saude.gov.br/bvs/publicacoes/pnad_panorama_saude_brasil.pdf >. Acesso em: 10 de março de 2019.

INOJOSA, R. M. Sinergia em Políticas e Serviços Públicos: Desenvolvimento Social com Intersetorialidade. Cadernos FUNDAP. $n^{\circ}$. 22, p.102-110, 2001. Disponível em:

$<$ https://www.pucsp.br/prosaude/downloads/bibliografia/sinergia_politicas_servicos_publicos .pdf>. Acesso em: 10 de dezembro de 2012.

MALTA, D. C. et al. A cobertura da Estratégia de Saúde da Família (ESF) no Brasil, segundo a Pesquisa Nacional de Saúde, 2013. Ciência e Saúde Coletiva, v. 21, n 2, p. 327-338, 2016. Disponível em: < http://www.scielo.br/pdf/csc/v21n2/1413-8123-csc-21-02-0327.pdf>. Acesso em: 12 de abril de 2019.

MÔRA, L. B. et al. O uso da territorialização para apoio ao planejamento das ações de uma unidade de saúde da família. 2013. Disponível em:

<http://www.politicaemsaude.com.br/anais/trabalhos/publicacoes/198.pdf>. Acesso em: 08 de março de 2019.

OBSERVATÓRIO DE ANÁLISE POLÍTICA EM SAÚDE. Atenção primária à saúde em 2016: avanços desafio e resultados. 2017. Disponível em:

<https://analisepoliticaemsaude.org/oaps/noticias/58f51333a319ff1adc256e5205e4e372/>.

Acesso em: 12 de abril de 2019.

OLIVEIRA, E. B. A interdisciplinaridade na perspectiva de integrar as disciplinas da área de ciências da natureza e matemática. 2016. 224 f. Dissertação (Mestrado): Programa de Pós Graduação em Ensino na Educação Básica da Universidade Federal do Espírito Santo. Universidade Federal do Espírito Santo Centro. Espírito Santo - ES. 2016.

PESSOA, L. R. Mergulho em Montes Claros: desafios da alocação de recursos na rede SUS. 2005. 195 f. Tese (Doutorado): Escola Nacional de Saúde Pública, Planejamento e Gestão de Sistemas em Serviços de Saúde. Rio de Janeiro. 2005.

ROSA, T. C. S.; SAES, S. G.; ABULEAC, F.L. A Constituição de 1988 e as políticas públicas em saúde no Brasil. Revista de gestão em sistema de saúde. Revista de Gestão em Sistemas de Saúde - RGSS, São Paulo, v. 1, n. 1, p. 35-49, jan./jun. 2012. Disponível em: Revista de Gestão em Sistemas de Saúde - RGSS, São Paulo, v. 1, n. 1, p. 35-49, jan./jun. 2012. Disponível em: 
<http://www.revistargss.org.br/ojs/index.php/rgss/article/view/9/71>. Acesso em: 10 de novembro de 2019.

SILVA JUNIOR, E. S. et al. Acessibilidade geográfica à atenção primária à saúde em distrito sanitário do município de Salvador, Bahia. Revista brasileira de saúde materno-infantil, Recife, v. 10, supl. 1, p. 549-560, nov. 2010. Disponível em:

<http://www.scielo.br/pdf/rbsmi/v10s1/05.pdf>. Acesso em: 12 de março de 2019.

SILVA, S. P. A abordagem territorial no planejamento de políticas públicas e os desafios para uma nova relação entre estado e sociedade no Brasil. Cadernos gestão pública e cidadania, São Paulo, v. 17, n. 60, Jan./Jun. 2012.

SILVA, C. R. et al. Dificuldade de acesso a serviços de média complexidade em municípios de pequeno porte: um estudo de caso. Revista Ciência \& Saúde Coletiva. Volume 22, n⿳⺈ 4 , p. 1109-1120, 2017. Disponível em:

<https://www.scielosp.org/pdf/csc/2017.v22n4/1109-1120/pt>. Acesso em 12 de dezembro de 2019.

SCHERER, M. D. A.; Pires, D. Interdisciplinaridade: processo complexo de conhecimento e ação. Revista Tempus Actas de Saúde Coletiva. v. 5, no 1. p. 2011: 69-84. Disponível em: <http://www.tempusactas.unb.br/index.php/tempus/article/view/919/929> Acesso: 14 de dezembro de 2019.

SOUZA, C. Políticas públicas: uma revisão de literatura. Revista de Sociologias. Porto Alegre, ano 8, n. 16, jul/dez 2006, p. 20-45. Disponível em:

<http://www.scielo.br/pdf/soc/n16/a03n16>. Acesso em: 20 de abril de 2019.

SOUSA, M. F; HAMANN, E. M. Programa de Saúde da Família no Brasil: uma agenda incompleta? Ciência e Saúde Coletiva, v.14, supl.1, setembro/outubro 2009. Disponível em: <http://wsXzww.scielo.br/pdf/csc/v14s1/a02v14s1.pdf>. Acesso em: 28 de março de 2019.

WARSCHAUER, M.; CARVALHO, Y, M. O conceito "Intersetorialidade": contribuições ao debate a partir do Programa Lazer e Saúde da Prefeitura de Santo André/SP. Revista Saúde Soc. São Paulo, v.23, n.1, p.191-203, 2014. Disponível em:

<http://www.scielo.br/pdf/sausoc/v23n1/0104-1290-sausoc-23-01-00191.pdf>. Acesso em 10 de janeiro de 2019.

Data de recebimento: $26 / 12 / 2019$

Data de aceite: 09/06/2020

\section{Sobre os autores:}

Gláucio Jorge de Souza é Doutorando em Planejamento Urbano e Regional pela Universidade do Vale do Paraíba (UNIVAP) com início 2017, Mestre em Desenvolvimento Humano pela UNITAU ano de conclusão (2015), Pós Graduação Lattes Senso em Cuidados Críticos /Cardiologia - Universidade do Vale do Paraíba UNIVAP (2008) e Docência para Enfermeiros pela Faculdade da Aldeia de Carapicuíba FALC (2009), Graduação em Enfermagem Bacharel - Faculdades Integradas Teresa D’Ávila (2006). Atualmente atua como Docente e Responsável Técnico no SENAC e na Graduação de Enfermagem da Faculdade de Educação de 
Guaratinguetá (FACEG), ministrando aulas teóricas e práticas assistências. Possui experiência nas áreas Assistenciais em Atenção Básica a Saúde, Unidade de Terapia Intensiva (UTI), Pronto Socorro, Clínica Médica, Atendimento aos pacientes Críticos no intra e no pré hospitalar, Cardiologia, Docência, Gerenciamento e Responsabilidade Técnica de Serviços de Saúde. Endereço Eletrônico: glauciojsouza123@gmail.com

Cilene Gomes possui graduação em Arquitetura e Urbanismo pela Universidade de São Paulo (1983), mestrado e doutorado em Geografia Humana pela Universidade de São Paulo (1994, 2001). Estágios de pós-doutorado concluídos no Instituto de Pesquisa e Planejamento da Universidade Federal do Rio de Janeiro (2011), no Instituto de Psicologia da Universidade de São Paulo (2014) e no Programa de Pós-Graduação em Planejamento Urbano e Regional (PLUR) da Universidade do Vale do Paraíba (2017). Atualmente é docente-pesquisador do PLUR da Universidade do Vale do Paraíba e é coordenadora do Observatório da Região Metropolitana do Vale do Paraíba e Litoral Norte, sediado no Núcleo de Estudos do Espaço Urbano e Regional (NEUR). Tem experiência no campo do ensino e pesquisa na área de Arquitetura e Urbanismo, Geografia Humana e Planejamento Urbano e Regional, atuando principalmente nos seguintes temas: estudos urbanos e regionais, planejamento urbano e regional, urbanização, metropolização e regionalização, rede urbana, cidades, sociedade da informação e comunicação, geotecnologias, participação social, cidadania, metodologia científica e psicologia social. Endereço Eletrônico: cilenegomes2011@ gmail.com.

Valéria Zanetti possui graduação em História pela Universidade Federal de Ouro Preto (1988), mestrado em História Social pela Pontifícia Universidade Católica do Rio Grande do Sul (1994) e doutorado em História Social pela Pontifícia Universidade Católica de São Paulo (2008). Atualmente é professora dos cursos de licenciatura em História e Geografia e do Programa de Mestrado e Doutorado em Planejamento Urbano e Regional da Universidade do Vale do Paraíba (UNIVAP). Tem experiência na área de História, com ênfase em História do Brasil (Colonia e Império), Teoria da História, HIstória Contemporânea, Identidade, memória, Paleografia, escravidão e resistência. É membro do Comitê de Ética da UNIVAP, coordenadora do Núcleo de Pesquisa Pro-Memória São José dos Campos, do Centro de Memória e História da Universidade do Vale do Paraíba, membro do Conselho Municipal do Patrimônio Histórico, Artístico e Cultural (COMPHAC) e participa do Observatório da Paisagem (IPHAN, UNIVAP, UNITAU). Endereço Eletrônico: vzanetti@ univap.br. 